\title{
Aspectos técnicos e biológicos da obtençãode oócitos bovinos: \\ revisão de literatura
}

\section{Technical and biological aspects on recovery of oocytes from cattle donors: bibliographical review.}

\author{
Marcelo Marcondes Seneda ${ }^{1}$; Cesar Roberto Esper ${ }^{2}$; \\ Joaquim Mansano Garcia ${ }^{3}$; Evelyn Rabelo Andrade ${ }^{4}$
}

Resumo

Desde o primeiro relato de colheita de embriões, um grande número de trabalhos tem apresentado os esforços para melhorar a contribuição genética de fêmeas bovinas. O objetivo desta revisão consiste em relacionar os primeiros trabalhos, novas descobertas e inovações técnicas da obtenção de oócitos em vacas.

Palavras chave: Oócitos, embriões, bovinos.

\begin{abstract}
Since the first work about embryo collection, a great amount of research and development has founded on enhancing genetic contribution of cows. The aim of this publication is to give an account of the current knowledge, new findings and techniques on recovery of oocytes from cattle donors.
\end{abstract}

Key words: Oocytes, embryos, cattle.

\section{Introdução e Revisão de Literatura}

As modificações na reprodução dos animais possivelmente ocorrem desde sua domesticação, uma vez que o cativeiro por si próprio já seria capaz de alterar o comportamento reprodutivo. Atualmente o homem tem realizado esforços para incrementar a produtividade na exploração das espécies domésticas, e isto tem sido verificado de diversas formas.
Modificações na nutrição, instalações, manejo sanitário e reprodutivo têm sido pesquisadas há várias décadas (BOLS et al., 1997)

As fêmeas bovinas também têm sido alvo de várias pesquisas, para que o aproveitamento mais racional de seus gametas seja possível. Segundo Hafez e Hafez (2000), a fêmea bovina possui, por ocasião da puberdade, aproximadamente 70.000 oócitos em

\footnotetext{
${ }^{1}$ Marcelo Marcondes Seneda ${ }^{1}$ Prof. Adjunto e Reprodução e Obstetrícia de Grandes Animais Depto de Clínica Veterinária, CCA/UEL. Email: mseneda@uel.br

2 Cesar Roberto Esper - Prof. Adjunto/ FCAV-UNESP, Jaboticabal, SP

3 Joaquim Mansano Garcia - Prof. Assist. Doutor/ FCAV-UNESP, Jaboticabal, SP

4 Evelyn Rabelo Andrade - Doutoranda, UECE, Fortaleza, CE.

* Autor para correspondência
} 
seus ovários, que pelas vias naturais, podem gerar perto de $0,01 \%$ de produtos viáveis, ou seja, um número próximo a dez descendentes em toda sua vida reprodutiva (BOLS et al., 1997).

Dentre as alternativas propostas para um maior aproveitamento dos gametas de vacas e novilhas, destaca-se a transferência de embriões (TE), que vem sendo usada com sucesso desde 1951, quando Willet et al. (1951) fizeram o primeiro relato de um produto nascido por esta técnica. Sendo inicialmente um procedimento cirúrgico, a TE posteriormente foi adaptada para uma técnica não invasiva (GREVE, 1977), sendo assim utilizada até os dias atuais. Em um programa de TE, cada doadora pode multiplicar em mais de três vezes o número de descendentes de sua vida reprodutiva (BOLS et al., 1997).

No entanto, a TE apresenta algumas restrições. Relatos de cistos ovarianos, endometrites e lesões iatrogênicas foram observados em grande número de doadoras (BAK; GREVE; SCHMIDT et al., 1989). Kruip et al. (1991) observaram que o número de estruturas recuperadas nem sempre justificava a realização da técnica. Meintjes et al. (1995) relataram que a contínua interrupção do ciclo estral e as terapias gonadotróficas poderiam comprometer a fertilidade da doadora e Bols et al. (1997) ressaltaram a variabilidade de respostas ao estímulo gonadotrófico, além da regular colheita de estruturas não fecundadas. Outro aspecto é que a colheita de embriões mostra-se inviável em algumas situações de infertilidade extra-ovariana, como hidrosalpinge bilateral (BOLS; VAN SOON; KRUIF et al., 1996b) e nas obstruções uterinas (SENEDA et al., 2000). Uma limitação adicional diz respeito à difícil aplicabilidade da TE em programas de biotecnologia, como a clonagem, que requer embriões em estádios iniciais de desenvolvimento (LAMBERT et al., 1986).

Embora a TE continue sendo amplamente utilizada, o aproveitamento dos oócitos bovinos ganhou novo impulso em 1982, quando Brackett et al. (1982) relataram o nascimento do primeiro bezerro obtido pela produção in vitro (PIV) de embriões. A PIV de embriões, a partir de oócitos de matadouro, tem sido utilizada desde então com perspectivas promissoras, tendo viabilizado nascimento de expressivo número de produtos (GALLI; LAZZARI; 1996).

Apesar da possibilidade de se aumentar o número de descendentes de doadoras que vieram ao óbito, a PIV de embriões a partir de oócitos de matadouro possui limitações, como o tempo de transporte do matadouro ao laboratório, o desconhecimento acerca do estado de saúde, do padrão hormonal dos animais e, principalmente, a impossibilidade de repetição da técnica para um mesmo animal, já que os ovários são obtidos com o óbito do mesmo. Segundo Pieterse et al. (1988), a recuperação de oócitos é a base do programa de fecundação in vitro (FIV) e com as limitações dos oócitos obtidos de matadouro, alternativas foram propostas para o aproveitamento de oócitos de animais vivos (BOLS et al., 1995; BROGLIATTI; ADAMS, 1996).

Uma técnica que permite o aproveitamento de oócitos de animais vivos é a laparoscopia, podendo ser transvaginal (REINCHENBACH et al., 1993) ou paralombar (LAMBERT et al., 1983). Consistindo de um procedimento cirúrgico menos cruento que a laparatomia, a laparoscopia permitiu a recuperação de oócitos em novilhas (LAURINCÍK et al., 1991), e mesmo em bezerras de apenas 3 semanas de idade (ARMSTRONG et al., 1994). Apesar das perspectivas promissoras, limitações para a laparoscopia foram listadas por Looney et al. (1994). Estes autores comentaram ser a técnica trabalhosa, além da ocorrência de fibroses e aderências ovarianas após sua realização.

Outra técnica proposta para a recuperação de oócitos é citada por Hinrichs, Kenney e Kenney (1990). Trata-se da colpotomia, na qual a mão do operador manipula os ovários através de uma incisão intravaginal, pressionando-os contra a parede abdominal, por onde uma agulha para a punção folicular é introduzida. Mas os riscos de peritonite e evisceração através da incisão vaginal limitaram a expansão deste procedimento. 
Valendo-se das possibilidades da ultra-sonografia, Callesen, Greve; Christensen (1987) relataram pela primeira vez o uso desta técnica para a obtenção de oócitos bovinos. Através da manipulação transretal, os ovários foram posicionados dorso-lateralmente na cavidade abdominal, viabilizando sua visualização com um transdutor de $3.5 \mathrm{MHz}$ posicionado sobre a pele, na região da fossa paralombar, local de introdução da agulha capaz de puncionar os folículos. Apesar do êxito deste procedimento, a recuperação de oócitos bovinos com uso da ultra-sonografia só ganhou grande impulso um ano mais tarde, com nova modificação que tornou o procedimento de mais fácil e rápida aplicação. Pieterse et al. (1988) alteraram a técnica descrita para humanos (FEICHTINGER; KEMETER, 1986) e descreveram a aspiração folicular via transvaginal através da ultrasonografia, que tornou viável o aproveitamento de oócitos bovinos, sem as limitações dos procedimentos existentes até então.

Após avaliações, a técnica mostrou-se simples e inócua, viabilizando a recuperação, maturação e fecundação de oócitos imaturos, podendo ser repetida várias vezes em um mesmo animal (KRUIP et al., 1991; PIETERSE et al., 1991; BOLS et al., 1995a; MEINTJES et al., 1995, BOLS et al., 1996), inclusive com um aumento no número de folículos após várias semanas de aspirações foliculares (STUBBINGS ; WALTON; 1995).

A possibilidade de obtenção de embriões em situações de infertilidade extra-ovariana motivou vários grupos à realização do procedimento, tendo-se verificado nascimento de diversos produtos (LOONEY et al., 1994; HASLER et al., 1995; BOLS; VAN SOON; KRUIF, 1996b; SENEDA et al., 2000), confirmando as observações de Dawson (1977), a respeito de que a maioria das fêmeas bovinas classificadas como inférteis mostrava-se capaz de produzir gametas viáveis. Se a aspiração folicular apresentou-se viável para fêmeas com limitações reprodutivas, sua aplicação mostrou-se mais ampla quando utilizada em fêmeas saudáveis, produzindo quatro vezes mais embriões em relação à TE (KRUIP et al., 1994), embora com maior custo por embrião (RODRIGUES; GARCIA, 2000).

Para reduzir os custos pertinentes aos procedimentos in vitro, diversos trabalhos foram descritos com o intuito de incrementar a técnica. Segundo BOLS et al. (1997) os principais fatores a serem analisados reúnem-se nos aspectos biológicos e os de ordem técnica, que serão abordados a seguir.

As variáveis técnicas constituem o método de obtenção dos oócitos e este possui considerável impacto sobre a quantidade e a morfologia dos complexos cumulus oophorus, e conseqüentemente sobre a competência para o desenvolvimento (BOLS et al., 1997). O tipo de agulha foi estudado por Bols et al. (1995), que propuseram a substituição das agulhas longas, específicas para aspiração folicular, por agulhas hipodérmicas descartáveis. Segundo estes autores, as agulhas descritas inicialmente apresentavam custo elevado e perdiam o gume rapidamente, prejudicando a recuperação dos oócitos. Posteriormente, Bols et al. (1997) mostraram que agulhas hipodérmicas descartáveis de 18 e 19 Gauge (G) permitiam boa taxa de recuperação, preservando a qualidade dos oócitos. Diâmetros maiores que $19 \mathrm{G}$ relacionavam-se a maiores taxas de recuperação embora com maior percentual de oócitos desnudos, enquanto agulhas de menor diâmetro apresentavam índices reduzidos de recuperação de oócitos. No entanto, o sistema que viabiliza o uso de agulhas descartáveis possui maior número de conexões, obstáculos capazes de reter os oócitos (BOLS et al., 1997).

Estreitamente relacionado com a agulha utilizada encontra-se a pressão de vácuo (BOLS et al., 1996a). Estes autores relacionaram que baixas pressões, como $50 \mathrm{~mm} \mathrm{Hg}$, foram pouco eficientes para a aspiração, enquanto que pressões maiores como $120 \mathrm{~mm} \mathrm{Hg}$ danificavam o revestimento do cumulus oophorus. Há uma grande variação entre os trabalhos, com valores de 40 a $400 \mathrm{~mm} \mathrm{Hg}$ (BOLS et al., 1996), embora isto deva ser considerado com reservas, já que todo o sistema (comprimento e diâmetro de conexões, altura do equipamento de vácuo, diâ- 
metro da agulha) pode influenciar na pressão de vácuo final. Para quantificar a pressão negativa de forma mais real, estes autores sugeriram mensurar o vácuo em volume de água por minuto. Mesmo assim, há variações consideráveis, de 4,4 a $40 \mathrm{ml}$ de água/minuto (PIETERSE et al., 1988; VOS et al., 1994; BUNGARTZ et al., 1995; RICK et al., 1996).

O comprimento do bisel da agulha e a taxa de recuperação de oócitos foram analisados por BOLS et al. (1997). Estes autores acreditavam que o bisel curto seria mais eficiente, pela maior facilidade de ser introduzido rapidamente no interior do folículo. Contrariando as expectativas, os autores relataram melhor recuperação e qualidade de oócitos quando agulhas de bisel longo foram utilizadas.

A frequiência do transdutor constitui-se em variável importante no processo de recuperação dos oócitos (HASHIMOTO et al., 1999a). Há citações de freqüências de $3.5 \mathrm{MHz}$ (CALLENSEN et al., 1987), 7.5 MHz (VAN DER SCHANS et al., 1991), 5.0 MHz (HASLER et al., 1995) e $6.5 \mathrm{MHz}$ (BUNGARTZ et al., 1995). A maioria dos autores cita a utilização de transdutores convexos ou setoriais para a aspiração folicular transvaginal (LOONEY et al., 1994; MEINTJES et al., 1995; BOLS et al., 1996; BOLS et al., 1998; FRY et al., 1998; CARLIN et al., 1999), com poucos relatos de transdutores lineares (HASHIMOTO et al., 1999; MATTHEWS; PETERSEN; VAN BEEDK, 1995). No entanto, o transdutor linear é bastante difundido na área de reprodução em grandes animais (SIANANGAMA; RAJAMAHENDRAN, 1996; BARUSELLI et al., 1997; FIGUEIREDO et al., 1997; LEYVA; BUCKRELL; WALTON, 1998; DICKIE et al., 1999) e a sua utilização para a aspiração folicular transvaginal poderia ser incrementada, uma vez que Hashimoto et al. (1999) obtiveram resultados satisfatórios com este equipamento.

Os aspectos biológicos da aspiração folicular possuem dados contraditórios, que podem ser considerados pela complexidade da questão, uma vez que Bols et al. (1997) propôs três níveis principais de variação: a importância da condição fisiológica da doadora; o quanto o folículo podia interferir na qualidade do oócito e o momento da seleção folicular.

A condição fisiológica da doadora, considerandose peso, raça, idade e a própria variação individual, tem merecido atenção em diversos artigos. Katska e Smorag (1984), trabalhando com ovários de matadouro, não encontraram diferenças na qualidade dos oócitos entre animais jovens (a partir de 18 meses) e senis (até 17 anos), embora tenham notado redução na produção de gametas nos animais mais velhos. Looney et al. (1994) relataram a possibilidade de aspiração folicular nos animais bastante jovens, e Brogliatti e Adams (1996) obtiveram oócitos de bezerras com apenas 6 semanas de idade com a utilização de um transdutor adequado. No entanto, os animais pré-púberes têm mostrado reduzida competência de seus oócitos para chegar até blastocisto (REVEL et al., 1995), embora Armstrong et al. (1994) tenham conseguido resultados animadores com bezerras de apenas três semanas de idade através do estímulo gonadotrófico. Parece haver um consenso quanto à condição corporal, em que animais subnutridos seriam doadores de oócitos com menor capacidade para desenvolverem-se até blastocisto (LOPEZ RUIZ et al., 1996), e há indícios de que animais submetidos à situações de estresse também seriam doadores de oócitos menos competentes (SENEDA et al., 2000a). Poucos estudos têm relatado variações entre as raças, sendo difícil a comparação entre os mesmos, pelas variações nos métodos utilizados.

A fase reprodutiva, incluindo o período gestacional, tem sido alvo de diversos estudos propostos para apontar um momento mais favorável à realização da aspiração folicular. Pelo fato do ciclo estral do bovino apresentar geralmente duas a três ondas de crescimento folicular (BUNGARTZ; NIEMANN, 1994; GINTHER, 2000), chegando mesmo a quatro e cinco ondas, sob tratamento com progestágenos (BOLS, 1997), a dinâmica folicular tem sido analisada na tentativa de se estabelecer relações que permitam melhor aproveitamento dos oócitos. Bols et al. (1997) observou que oócitos apre- 
sentavam diferentes capacidades de desenvolvimento, conforme a fase do ciclo estral em que foram colhidos. Um número maior de embriões foi obtido após cultivo de oócitos recuperados entre os dias 14 e 16 do ciclo estral, segundo Machatkova, Petilikova e Dvoracek (1995). No entanto, trabalhando com ovários de abatedouro, Smith et al. (1995) analisaram a dominância folicular, relatando que a produção in vitro de embriões não era afetada pela presença ou ausência do folículo dominante. Assey; Hyttel e Kanya (1994) analisaram a ultra-estrutura de oócitos de folículos dominantes e subordinados, e relataram degenerações prematuras no oócito dominante, embora Rhodes et al. (1997) não tenham constatado diferenças na competência para o desenvolvimento entre oócitos obtidos de folículos dominantes ou folículos subordinados. Estas observações foram corroboradas pelos resultados de Kruip et al. (1991) e Bungartz e Neimaet (1995), ao demonstrarem que blastocistos viáveis podiam ser obtidos independentemente da fase reprodutiva. Meintjes et al. (1995) constataram que vacas gestantes podiam ser submetidas à técnica durante o primeiro trimestre de gestação e, Sauvé (1998) até o sexto mês de prenhez, sem que fosse notado qualquer prejuízo à gestação ou às vacas.

As relações entre dinâmica e atresia foliculares também têm sido estudadas, com relatos de presença de oócitos saudáveis em folículos claramente atrésicos (KRUIP; DILEMAN, 1982; BLONDIN; SIRARD, 1995). Um leve grau de atresia folicular parece mesmo ter um efeito benéfico no oócito destinado para a FIV, segundo Wurth e Kruip (1992). Para estes autores, o efeito inibitório da maturação do oócito seria mais eficaz estando a parede folicular perfeitamente íntegra, enquanto o folículo levemente atrésico deixaria de exercer este efeito, favorecendo a maturação in vitro. Além disso, a competência do oócito seria adquirida tardiamente em relação ao desenvolvimento folicular (BLONDIN; SIRARD, 1995), o que justificaria a assincronia entre desenvolvimento folicular e oocitário. Uma vez caracterizada a atresia do oócito, então sua inviabilidade para o desenvolvimento in vitro seria compreendida, uma vez que De Loos et al. (1989) demonstraram pela análise ultraestrutural que as organelas agrupavam-se de forma compacta nos oócitos atrésicos, enquanto que nos oócitos de melhor qualidade apresentavam-se distribuídas de forma mais homogênea.

Mesmo diante da dificuldade de comparação dos parâmetros citados, o fator individual tem sido apontado como de grande importância na competência dos oócitos para o desenvolvimento, apesar das variações pertinentes à raça, idade e condição corporal (BOLS et al., 1997; WATANABE et al., 1999).

Além do fator individual, um outro aspecto de ordem biológica a ser considerado como possível variável na FIV é a interação folículo-oócito. A literatura é escassa quanto à influência do diâmetro do folículo sobre a qualidade e competência do oócito para o desenvolvimento, quando o oócito é obtido pela aspiração folicular guiada pela ultra-sonografia. Tem sido estudada a competência do oócito com aspectos indiretamente ligados ao diâmetro folicular, como a dominância folicular (SMITH et al., 1996; RHODES et al., 1997), a relação entre componentes do fluido e diâmetro folicular (STUBBINGS; WALTON, 1995) e interação entre diâmetro do folículo e qualidade do cumulus oophorus (FUKUI; SAKUMA, 1980).

Outros autores trataram diretamente do efeito do diâmetro do folículo sobre a competência do oócito para o desenvolvimento. Carolan et al. (1996) relataram que não há influência do tamanho do folículo sobre a maturação do oócito, embora Lonergan et al. (1994) tenham mostrado melhores índices de desenvolvimento de embriões oriundos de oócitos de folículos maiores de 6 milímetros ( $\mathrm{mm})$, quando comparados com oócitos de folículos menores e Tan e Lu (1990) estabelecendo três categorias de folículos, <2 mm, de 2- $6 \mathrm{~mm}$ e $>6 \mathrm{~mm}$, mostraram uma maior competência de desenvolvimento nos oócitos oriundos de folículos maiores de $2 \mathrm{~mm}$, sendo os $>6 \mathrm{~mm}$ melhores que os de 2 a $6 \mathrm{~mm}$. Pavlok, 
Lucans-Hans e Niemann (1992), relataram que oócitos de folículos médios (2-4mm) e grandes (4$8 \mathrm{~mm}$ ), apresentaram melhor desempenho na produção de blastocistos, em comparação a oócitos de folículos pequenos (1-2mm), resultados semelhantes aos encontrados por Blondin e Sirard (1995) e Seneda et al. (2001). Parece haver um consenso geral sobre os folículos de 1-2mm, cujos oócitos seriam de menor competência para os procedimentos de cultivo in vitro (BOLS et al., 1997). Porém, para a aspiração folicular transvaginal, são considerados folículos a partir de $2 \mathrm{~mm}$, em função da resolução do aparato de ultra-sonografia (PIERSON; GINTHER 1988; BUNGARTZ et al., 1995; MEINTJES et al., 1995).

Se a competência do oócito obtido pela aspiração folicular transvaginal parece não ser influenciada pelo tamanho do folículo, há evidências de que o mesmo não ocorra em relação à taxa de recuperação. Este parâmetro consiste no número de oócitos recuperados após punção de 100 folículos (PIETERSE et al., 1988). Apesar deste índice ser bastante difundido, Bols et al. (1996b) salientaram a importância de correlacionar a taxa de recuperação com o número de oócitos por sessão, uma vez que este último traduz o número real de estruturas disponíveis aos procedimentos de FIV. A taxa de recuperação pode ser influenciada pelas terapias gonadotróficas (PIETERSE et al., 1992; MEINTJENS, et al., 1995), freqüência de realização da técnica (GIBBONS et al. 1994), fase do ciclo (VOS et al. 1994), pressão de vácuo e tipo de agulha (BOLS et al., 1996b), tamanho do folículo (SENEDA et al., 2001), além da experiência do operador (GARCIA et al., 1998). Um ponto em comum destes artigos foi a menor recuperação de oócitos quando havia predomínio de grandes folículos no momento da aspiração, fato observado também em ovários de matadouro por Lonergan et al. (1994), ao constatarem taxa de recuperação maior quando os folículos aspirados eram pequenos (2 a $6 \mathrm{~mm}$ ) em comparação aos maiores de $6 \mathrm{~mm}$.
Pieterse et al. (1992) realizaram a aspiração folicular três dias após estímulo gonadotrófico com PMSG e relataram aumento do número e tamanho de folículos, e pior taxa de recuperação de oócitos, embora Looney et al. (1994) tenham relatado incremento no número de folículos e também na recuperação de oócitos, após estímulo gonadotrófico. Pieterse et al. (1991) e Goodhand et al. (1999) relataram melhor recuperação quando predominavam folículos pequenos no ovário e menos oócitos por sessão quando havia predomínio de folículos maiores que $6 \mathrm{~mm}$. Resultados análogos foram descritos por Seneda et al. (2001), ao compararem folículos < $4 \mathrm{~mm}$ e $>4 \mathrm{~mm}$. A relação inversa entre maior diâmetro folicular e taxa de recuperação de oócitos por sessão tem sido justificada de diversas formas, como alterações morfológicas no complexo cumulus oophorus (BOLS et al., 1998), viscosidade do fluido folicular (GOODHAND et al., 1999) e quantidade de material a ser aspirado e pressão intrafolicular (SENEDA, 1999), apesar do consenso entre estes autores quanto à maior facilidade de aspiração dos folículos maiores.

Assim, apesar dos folículos maiores que $5 \mathrm{~mm}$ serem aspirados mais facilmente (BOLS et al., 1997), a aspiração de folículos pequenos parece disponibilizar maior número de oócitos para o cultivo, sem prejuízo ao desenvolvimento in vitro até blastocisto. É evidente que apesar da menor recuperação verificada a partir dos folículos maiores, estes devam ser puncionados (BOLS et al., 1997). Desta forma, um aspecto a ser considerado seria a aspiração realizada em um momento que predominassem folículos de menor tamanho, conforme sugerido por Hashimoto et al. (1999) e Seneda (2001). Considerando a possibilidade de alteração do diâmetro folicular pelos estímulos hormonais, a modificação nos protocolos de terapias gonadotróficas poderia contribuir neste sentido. Outra possibilidade seria a restrição do crescimento folicular, por meio de sessões de aspiração com intervalos de três e quatro dias, conforme sugerido por Gibbons et al. (1994) 
quando demonstraram maior número de folículos recrutados após cada aspiração. Este procedimento mostrou-se interessante por dispensar gonadotrofinas exógenas, mas exigia o dobro de procedimentos para um mesmo número de folículos aspirados, quando em comparação ao estímulo hormonal (RODRIGUES; GARCIA; 1998; SAUVÉ, 1998).

Por esta razão, tem-se continuado a buscar protocolos com uso de gonadotrofinas. Além do FSH, o PMSG tem sido usado, principalmente em animais pré-púberes e com baixa resposta ao uso de FSH (ARMSTRONG et al., 1994). Apesar do maior número de folículos visualizados com o estímulo gonadotrófico, o número de oócitos por sessão mostrou-se proporcionalmente menor ao esperado (LOONEY et al., 1994; RODRIGUES; GARCIA, 1998; GOODHAND et al., 1999), possivelmente pelo maior diâmetro dos folículos no momento da aspiração (SENEDA et al., 2000a). Há considerável variação nos protocolos de estímulo hormonal precedendo a aspiração folicular. A literatura apresenta trabalhos preconizando o melhor dia de punção folicular como sendo quatro (BUNGARTZ; NEIMAN, 1995; GOODHAND et al., 1996), cinco (BORDIGNON et al., 1996; PUELKER et al., 1999) e mesmo seis dias (MEINTJES et al., 1995) após o início do FSH. Estes trabalhos apresentaram predominância de folículos grandes (até $19 \mathrm{~mm}$ ), sendo a maioria com diâmetro maior que $10 \mathrm{~mm}$.

Considerando essas informações, conclui-se que modificações de ordem técnica e biológica ainda são necessárias para aumentar a eficiência do procedimento e disponibilizar mais oócitos para os procedimentos de produção in vitro de embriões. $\mathrm{O}$ avanço das técnicas deve viabilizar a utilização de animais bastante jovens para diminuir o intervalo entre gerações, acelerar o incremento genético e o estudo da competência de oócitos para o desenvolvimento. Outras espécies devem ser contempladas além da bovina. O estudo de protocolos de estímulo gonadotrófico e melhorias na viabilidade dos embriões produzidos in vitro devem ser estimulados, bem como maior eficiência no procedimento de vitrificação de oócitos. O domínio dessas técnicas deve tornar a recuperação de oócitos e produção in vitro de embriões uma potencial alternativa para os programas clássicos de transferência de embriões, sem as limitações de custo existentes no momento.

\section{Referências}

ARMSTRONG, D. T. et al. Gonadotropins stimulations regimens for follicular aspiration and in vitro embryo productions from calf oocytes. Theriogenology, Stoneham, v. 42, p. 1227-1236, 1994.

ASSEY, R. J.; HYTTEL, P.; KANUYA, N. Oocyte structure in dominant and subordinate follicles in zebu cattle (Bos indicus). Anatomy and Embryology, Berlin, v.190, p.461-468, 1994.

BAK, A.; GREVE, T.; SCHMIDT, M. Effect of super ovulation on reproduction. Theriogenology, Stoneham, v.31, p. 169, 1989. (Abstract).

BARUSELLI, P. S. et al. Ovarian follicular dynamics during the estrous cycle in buffalo (Bubalus bubalis). Theriogenology, Stoneham, v.47, n.8, p.1531-1547, 1997.

BLONDIN, P.; SIRARD, M. D. Oocyte and follicular morphology as determining characteristics for developmental in bovine oocytes. Molecular Reproduction and Development, New York, v.41, p.5462, 1995.

BOLS, P. E. J. et al. Transvaginal ovum pick-up (OPU) in the cow: new disposable needle guindance system. Theriogenology, Stoneham v.43, p.677-687, 1995.

BOLS, P. E. J. et al. Effects of aspiration vacum and needle diameter on cumulus oocyte complex morphology and developmental capacity of bovine oocytes. Theriogenology, Stoneham, v.45, p.1001-1014, 1996.

BOLS, P. E. J. et al. Transvaginal oocyte pick-up in infertile Belgium Blue donor cows: preliminary results. Theriogenology, Stoneham, v.45, p.359, 1996a. (Abstract)

BOLS, P.E.J.; VAN SOON, A.; KRUIF, A. Gebruik van de transvaginale Ovum Pick-Up techniek: geboorte van de eerste OPU kalveren in België. Vlaams Diergeneeskunding Tijdschrift, Merelbeke, v.65, p.86-91, 1996b

BOLS, P. E. J. et al. Effects of needle tip bevel and aspiration procedure on the morphology and developmental capacity bovine compact cumulus oocyte complexes. Theriogenology, Stoneham, v.47, p.12211236, 1997. 
BOLS, P. E. J. Transvaginal ovum pick-up in the cow: technical and biological modifications. 1997. Thesis $(\mathrm{PhD})$ - Faculty of Veterinary Medicine, University of Ghent, Belgium, 1997a

BOLS, P. E. J. et al. Effects of long-term treatment with bovine somatotropin on follicular dynamics and subsequent oocyte and blastocyst yield in an OPU-IVF program. Theriogenology, Stoneham, v. 49, p. 983-995, 1998.

BORDIGNON, V. et al. Effect of GnRH injection on recovery rate, meiotic synchronization and developmental competence of oocytes aspirated from superestimulated heifers. Theriogenology, Stoneham, v.45, n.1, p.352, 1996. (Abstract).

BRACKETT, B.G. et al. Normal development following in vitro fertilization in the cow. Biology of Reproduction, Champaign, v.101, p.147-158, 1982.

BROGLIATTI, G. M., ADAMS, G. P. Ultrasound-guided transvaginal oocyte collection in prepurbetal calves. Theriogenology, Stoneham, v.45, p.1163-1176, 1996.

BUNGARTZ, L.; NIEMAN, H. Assesment of the presence of a dominant follicle and selection of dairy cows suitable for superovulation by a single ultrasound examination. Journal of Reproduction and Fertility, Cambridge, v.101, p.583-591, 1994.

BUNGARTZ, L. et al. Collection of oocytes from cattle via follicular aspiration aided by ultrasound with or without gonadotropin pretreatment and in different reproductive stages. Theriogenology, Stoneham, v.43, n.667-675, 1995.

CALlESEN, H.; GREVE, T.; CHRISTENSEN, F. Ultrasonically guided aspiration of bovine follicular oocytes. Theriogenology, Stoneham, v.27, p.217, 1987. (Abstract).

CARLIN, S. K. et al. Effects of ultrasound-guided transvaginal follicular aspiration on oocyte recovery and hormonal profiles before and after $\mathrm{GnRH}$ treatment. Theriogenology, Stoneham, v.51, p.1489-1503, 1999.

CAROLAN, C. et al. Effect of follicle size and quality on the ability of follicular fluid to support cytoplasmic maturation of bovine oocytes. Molecular Reproduction and Development, New York, v.43, p.477-483, 1996.

DAWSON, F. L. M. Reproductive potential in female cattle discarded as infertile. Journal of Reproduction and. Fertility, Cambridge, v.51, p.53-56, 1977.

DELOOS, F. et al. Morphology of immature bovine oocytes. Gamete Research, New York, v.24, p.197-204, 1989.

DICKIE, M. C. et al. Determination of corpora lutea numbers in Booroola - Texel ewes using transrectal ultrasound. Theriogenology, Stoneham, v.51, n.7, p.12091224, 1999.
FEICHTINGER, W.; KEMETER, P. Transvaginal sector scan sonography for needle guided transvaginal follicle aspiration and other applications in gynecologic routine and research. Fertility and Sterility, Birmingham, v.45, p.722-725, 1986.

FIGUEIREDO, R. A. et al. Ovarian follicular dynamics in Nelore breed (Bos indicus) cattle. Theriogenology, Stoneham, v.47, n.8, p.1489-1505, 1997.

FUKUI, Y.; SAKUMA, Y. Maturation of bovine oocytes cultured in vitro: relation to ovarian activitty, follicular size and the presence or absence of cumulus cells. Biology of Reproduction, Champaign, v.22, p.669-673, 1980.

GALLI, C.; LAZZARI, G. Practical aspects of IVM/IVF in cattle. Animal Reproduction Science, Amsterdam, v.42, p.371-379, 1996.

GARCIA, J.M. et al. In vitro production (IVP) of bovine embryos: different procedures. Arquivos da Faculdade de Veterinária UFRGS, Porto Alegre, v.26, n.1, p.281, 1998.

GIBBONS, J. R. et al. Effects of once- versus twiceweekly transvaginal follicular aspiration on bovine oocyte recovery and embryo development. Theriogenology, Stoneham, v.42, p.405-419, 1994.

GINTHER, O. J. Selection of the dominant follicle in cattle and horses. Animal Reproduction Science, Amsterdam, v.60-61, p. 61-79, 2000.

GOODHAND, K.L. et al. In vivo oocyte recovery and in vitro embryo production in cattle pre-treated with FSH, progestogen and estradiol. Theriogenology, Stoneham, v.45, n.1, p.355, 1996. (Abstract).

GOODHAND, K. L. et al. In vivo oocyte recovery and in vitro embryo production from bovine donors aspirated at different frequencies or following fsh treatment. Theriogenology, Stoneham, v.51, p.951-961, 1999.

GREVE, T.; LEHN-JENSEN, H.; RASBECH, N.O. Nonsurgical recovery of bovine embryos. Theriogenology, Stoneham, v.7, n.4, p.239-250, 1977.

HAFEZ, E. S. E.; HAFEZ, B. Folliculogenesis, Egg Maturation, and Ovulation. In:__. Reproduction in farm animals. Philadelphia: Lippincott Williams \& Wilkins, 2000. Cap.5, p.68-82,

HASHIMOTO, S. et al. Ultrasound-guided follicle aspiration: effect of the frequency of a linear transvaginal probe on the collection of bovine oocytes. Theriogenology, Stoneham, v.52, p.131-138, 1999a.

HASLER, J. F. et al. Production, freezing and transfer of bovine IVF embryos and subsequent calving results. Theriogenology, Stoneham, v.43, p.141-152, 1995. 
HINRICHS, K.; KENNEY, D. F.; KENNEY, R. M. Aspiration of oocytes from mature and immature preovulatory follicles in the mare. Theriogenology, Stoneham,v.34, p.107-112, 1990.

KATSKA L; SMORAG, Z. Number and quality of oocytes in relation to age of cattle. Animal Reproduction Science, Amsterdam, v.7, p.451-460, 1984.

KRUIP, T. A.M.; DIELEMAN, S. J. Macroscopic classification of bovine follicles and its validation by micromorphological and steroid biochemical procedures. Reproduction, Nutrition and Development, Paris, v.22, p.465-473, 1982.

KRUIP, T. A. et al. A new method for bovine embryo production: a potential alternative to superovulation. Veterinary Record, Lonon, v.128, p. 208-210, 1991.

KRUIP, T. A. M. et al. Potential use of ovum pick-up for embryo production and breeding in cattle. Theriogenology, Stoneham, v.42, p.675-684, 1994.

LAMBERT, R. D. et al. Endoscopy in cattle by the paralumbar route: technique for ovarian examination and follicular aspiration. Theriogenology, Stoneham, v.20, n.2, p.149-161, 1983.

LAMBERT, R. D. et al. In vitro fertilization of bovine oocytes matured in vivo and collected at laparoscopy. Theriogenology, Stoneham, v.25, n.1, p.117-133, 1986.

LAURINCÍK, J. et al. Timing of laparoscopies aspiration of preovulatory oocytes in heifers. Theriogenology, Stoneham, v.35, n.2, p.415-423, 1991.

LEYVA, V.; BUCKRELL, B. C.; WALTON, J. S. Regulation of follicular activity and ovulation in ewes by exogenous progestagen. Theriogenology, Stoneham, v.50, n.3, p. 395-416, 1998.

LONERGAN, P. et al. Effect of follicle size on bovine oocyte quality and developmental competence following maturation, fertilization, and culture in vitro. Molecular Reproduction and Development, New York, v.37, p.4853, 1994.

LOONEY, C. R. et al. Commercial aspects of oocyte retrieval and in vitro fertilization (IVF) for embryo production in problem cows. Theriogenology, Stoneham, v.41, p.67-72, 1994.

LOPEZ RUIZ, L. et al. Effect of body condition on the developmental competence of IVM/IVF bovine oocytes. Theriogenology, Stoneham, v.45, p.292, 1996. (Abstract).

MACHATKOVA, J. E.; PETILIKOVA, J.; DVORACEK, V. Developmental competence of bovine embryos derived from oocytes collected at various stages of the estrous cycle. Theriogenology, Stoneham, v.44, p.801-810, 1995.
MATTHEWS, L.; PETERSEN, H.; VAN BEEK, K. Use of linear ultrasound transducer for commercial application of transvaginal oocyte recovery. Theriogenology, Stoneham, v.43, p.275, 1995. (Abstract).

MEINTJES, M. et al. Transvaginal aspiration of oocytes from hormone-treated pregnant beef for in vitro fertilization. Journal of Animal Science, Champaign, v.73, p.967-974, 1995.

PAVLOK, A.; LUCANS-HAHN; NIEMANN, H. Fertilization and developmental competence of bovine oocytes derived from different categories of antral follicles. Molecular Reproduction and Development, New York, v.31, p.63-67, 1992.

PIERSON, R. A.; GINTHER, O. J. Ultrasonic imaging of the ovaries and uterus in cattle. Theriogenology, Stoneham, v.29, n.1, p.21-37, 1988.

PIETERSE, M. C. et al. Aspiration of bovine oocytes during transvaginal ultrasound scanning of the ovaries. Theriogenology, Stoneham, v.30, n.4, p.751-762, 1988.

PIETERSE, M. C. et al. Transvaginal ultrasound guided follicular aspiration of bovine oocytes. Theriogenology, Stoneham, v.35, n.1, p.19-24, 1991.

PIETERSE, M.C. et al. Repeated transvaginal ultrasoundguided ovum pick-up in ECG-treated cows. Theriogenology, Stoneham, v.37, p.273, 1992. (Abstract).

PUELKER, R. Z. et al. Avaliação do método de sincronização da onda folicular de vacas submetidas a estimulação com FSH para a produção in vitro de embriões. Arquivos da Faculdade de Veterinária UFRGS, Porto alegre, v. 27, n.1, p. 278, 1999. (Resumo).

REICHENBACH, H .D. et al. Transvaginal laparoscopic guided aspiration of bovine follicular oocytes : preliminary results. Theriogenology, Stoneham, v. 39, p. 295, 1993. (Abstract).

REVEL, F. L. et al. Low developmental capacity of in vitro matures and fertilized oocytes from calves compared with that of cows. Journal of Reproduction and Fertility, Cambridge, v.103, p.115-120, 1995

RHODES, F. M. et al. Bovine ovarian follicle and oocyte characteristics after emergence of the first follicular wave. Theriogenology, Stoneham, v.47, n.1, p.149, 1997. (Abstract).

RICK, G. et al. Long-term ultrasound guided ovum pickup in heifers from 6 to 15 months of age. Theriogenology, Stoneham, v.45, n.1, p.356, 1996. (Abstract).

RODRIGUES, C. F. M.; GARCIA, J. M. The application of ultrasound guided follicular aspiration in cattle. In: REUNIÃO ANUAL DA SBTE, 13., 1998, Atibaia. Anais... Atibaia: SBTE, 1998. p.156-159 
RODRIGUES, C. F. M.; GARCIA, J. M. Fecundação in vitro: aplicação comercial. Arquivos da Faculdade de Veterinária UFRGS, Porto Alegre, v.28, n.1,p. 186-187, 2000.

SAUVÉ, R. Ultrasound guided follicular aspiration and in vitro fertilization. In: . In: REUNIÃO ANUAL DA SBTE, 13., 1998, Atibaia. Anais... Atibaia: SBTE, 1998. p.141-145.

SENEDA, M. M. Aspiração folicular transvaginal guiada pela ultra-sonografia: efeito do diâmetro do folículo sobre a recuperação, qualidade e competência do oócito para o desenvolvimento in vitro. 1999. Dissertação (Mestrado em Patologia Animal) - Faculdade de Ciências Agrárias e Veterinárias, Uiversidade Estadual Paulista, Jaboticabal.

SENEDA, M. M. et al. Obtenção de embriões bovinos em um caso de obstrução uterina. Arquivos da Faculdade de Veterinária UFRGS, Porto Alegre, v.28, n.1, p.331, 2000.

SENEDA, M. M. et al. Effect of follicle size on recovery, quality, and developmental competence of oocytes obtained in vitro. In: INTERNATIONAL CONGRESS ON ANIMAL REPRODUCTION, 14., 2000, Stockholm. Proceeding... Stockholm: [s.n.], 2000a. v.1, p.62-63

SENEDA, M. M.et al. Relationship between follicle size and ultrasound-guided transvaginal oocyte recovery. Animal Reproduction Science, Amsterdam, v.67, p.3743, 2001.

SIANANGAMA, P. C.; RAJAMAHENDRAN R. Effect of hCG administration on day 7 of the estrous cycle on follicular dynamics and cycle length in cows. Theriogenology, Stoneham, v.45, n.3, p.583-592, 1996.
SMITH, L. C. et al. Oocyte quality in small antral follicles in the presence or absence of a large dominant follicle in cattle. Journal of Reproduction and Fertility, Cambridge, v.106, n. 193-199, 1996.

STUBBINGS, R. B.; WALTON, J. S. Effect of ultrasonically-guided follicle aspiration on estrous cycle and follicular dynamics in holstein cows. Theriogenology, Stoneham, v.43, p.705-712, 1995.

TAN, S. J.; LU, K. H. Effects of different oestrus stages of ovaries and sizes of follicles on generation of bovine embryos in vitro. Theriogenology, Stoneham, v.33, n.1, p.335, 1990. (Abstract).

VAN DER SCHANS, A. et al. Ultrasound-guided transvaginal collection of oocytes in the cow. Theriogenology, Stoneham, n.35, p.288, 1991. (Abstract).

VOS, P. L. A. M. et al. Evaluation of transvaginal ultrasound-guided follicle puncture to collect and follicular fluids at consecutive times relative to the preovulatory LH surge in eCG/PG treated cows. Theriogenology, Stoneham, v.41, p.829-840, 1994.

WATANABE, M. R. et al. A influência da doadora em programas OPU-FIV. Arquivos da Faculdade de Veterinária UFRGS, Local, v.27, n.1, p.307, 1999. (Resumo).

WILLET, E. L. et al. Successful transplantations of fertilized bovine ovum. Science, v. 113, p.247, 1951

WURTH, U.A.; KRUIP, T.A.M. Bovine embryo production in vitro after selection of the follicles and oocytes. . In: INTERNATIONAL CONGRESS ON ANIMAL REPRODUCTION, 12., 1992, The Ague. Proceeding... The Ague: [s.n.], 1992. v.1, p.387-379 